\title{
Minimally Invaded Sentinel Lymph Node Model for the Development of Intraoperative Infrared Fluorescence Imaging
}

\author{
Badiane Serigne Moussa1 ${ }^{*}$, Raes Florian ${ }^{2}$, Julien Sobilo², Natkunarajah Sharuja², \\ Lerondel Stéphanie ${ }^{2}$, Le Pape Alain² \\ ${ }^{1}$ UFR des Sciences de la Santé, Université Gaston BERGER, Saint-Louis, Sénégal \\ ${ }^{2}$ Centre for Small Animal Imaging, PHENOMIN-TAAM CIPA, CNRS UPS44, Orléans, France \\ Email: semobadiane@yahoo.fr, florian.raes@cnrs-orleans.fr, julien.sobilo@cnrs-orleans.fr, \\ sharuja.natkunarajah@cnrs-orleans.fr, stephanie.lerondel@cnrs-orleans.fr, alain.lepape@univ-tours.fr
}

How to cite this paper: Moussa, B.S. Florian, R., Sobilo, J., Sharuja, N., Stéphanie, L. and Alain, L.P. (2018) Minimally Invaded Sentinel Lymph Node Model for the Development of Intraoperative Infrared Fluorescence Imaging. Advances in Molecular Imaging, 8, 48-58.

https://doi.org/10.4236/ami.2018.84005

Received: July 10, 2018

Accepted: September 15, 2018

Published: September 18, 2018

Copyright (C) 2018 by authors and Scientific Research Publishing Inc. This work is licensed under the Creative Commons Attribution International License (CC BY 4.0).

http://creativecommons.org/licenses/by/4.0/

\begin{abstract}
In this study we implemented an axillary SLN invasion model to develop highly sensitive imaging strategies enabling detection of a very small amount of tumor cells. A highly diffusible molecular probe targeting $\alpha \mathrm{v} \beta 3$ and $\alpha \mathrm{v} \beta 5$ integrins was investigated either via IV or locoregional injections. We additionally documented the potential interferences of this Near Infrared Fluorescence Probe with Blue Patente V and ICG dyes routinely used to facilitate lymph node detection during surgery. The human mammary adenocarcinoma MDA-MB-231-luc model was injected into the forepaw of nude female rats to obtain a controlled invasion of the axillary LN. Thanks to its high sensitivity, BLI was selected to achieve in vivo quantitation of tumor cells in SLNs and determine eligible animals for the study. NIRF of integrins was performed at $680 \mathrm{~nm}$ both in vivo and ex vivo using spectral unmixing to suppress auto-fluorescence signal and preserve sensitivity. In vivo BLI was quite reliable in estimating discrete invasion by cancer cells in the LN with thresholds of detection and quantitation of about 500 and 1500 cells respectively. For fluorescence at $680 \mathrm{~nm}$, in vivo imaging is not suitable to detect micro-invasion, but ex vivo fluorescence with spectral unmixing of SLNs confirmed the presence of a tumor burden as low as 1500 cells expressing $\alpha v \beta 3 / \alpha v \beta 5$ integrins. Targeting few tumor cells inside a micro-invaded sentinel lymph node by molecular probes is not sensitive enough to provide direct in vivo or peroperative imaging. At the time NIRF is performed on the excised specimen, high sensitivity imaging associated with spectral unmixing allowed such detection within less than 1 minute of examination.
\end{abstract}

${ }^{\star}$ These authors contributed equally to this work. 


\section{Keywords}

Sentinel Lymph Node, Near Infrared Fluorescence Imaging, Photoacoustic Imaging, Molecular Imaging

\section{Introduction}

From the primary tumor, at the time tumor cells disseminate via lymph ducts, they accumulate in the first drainage site which is named the Sentinel Lymph Node (SLN). The interest in exploring the sentinel lymph node is to obtain information on the tumor invasion stage [1], because the invasion of SLNs by metastatic tumor is the major prognostic factor for patients with malignant tumors of epithelial origin [2]. In the clinical practice, the pathological examination of the excised specimen remains the gold standard for cancer confirmation. Apart from expensive fast molecular biology tests [3] [4], routine histopathology examinations require 4 - 7 days to detect minimal invasion and so does not allow the immediate assessment of the surgical outcome [5]. Positive micro-invasion of SLN in patients is relatively rare [6] [7] and the management of positive SLN in breast cancer has currently been revised. Most of the other SLN examinations are considered for gastric [8] [9], cervical, head and neck cancers [10] and melanoma [1]. Imaging strategies could provide information on the tumor invasion status faster than histopathology techniques. Nowadays, many different imaging agent sexist dedicated to the localization of LNs such as Blue Patente V (BPV) for visual direct observation or ICG (IndoCyanineGreen) for fluorescence peroperative imaging [11] [12]. For the imaging of invasion, labeled dedicated Monoclonal antibodies (MAbs) and RGD mimetic agents for targeting $\alpha \mathrm{V} \beta 3$ and $\alpha \mathrm{V} \beta 5$ integrins have been identified as efficient probes for in vivo imaging (PET, SPECT, MRI, NIRF). Targeting integrins from both tumor cells and tumor-related angiogenesis thank to low molecular weight highly diffusible probes should theoretically contribute to significantly increase the detection's sensitivity [13]. Among imaging modalities for such probes, Near Infrared Fluorescence Imaging (NIRF) is used more and more to investigate real-time peroperative visualization of tumors and SLNs [10] [14]. In this study, we performed preclinical investigations about assessment of tumor micro-invasion in a rat model of SLN. For such a purpose we considered Bioluminescence Imaging (BLI) thank to its very high sensitivity detecting a tiny number of tumor cells in order to select animals with a predefined SLN micro-invasion status. We then explored the ability of integrins NIRF to determine the invasion stage both in vivo and ex vivo on excised specimens. Considering the routinely used dyes such as Blue Patente V (BPV) by surgeons to evidence the SLNs, we investigated its potential quenching effect on fluorescence intensities from both an integrin targeting probe and ICG.

\section{Materials and Methods}

1) Ethics Statement 
All procedures on animals were performed in accordance with European ethical guidelines (European directives 2010/63/EU) and were approved by the Regional Committee for Animal Care and Ethics in Animal Experiments (CECCO $\left.\mathrm{n}^{\circ} 3\right)$.

\section{2) Cell Culture}

The MDA-MB-231-luc-D3H2LN human breast adenocarcinoma cancer cell line was obtained from Perkin Elmer (France). The cancer cell line was maintained according to the supplier's instructions.

3) Animals

Pathogen-free 8 to 12 week-old female nude rats were purchased from Taconic (Germany). Rats were acclimated for 7 days in the laboratory before experimentation and were maintained in sterilized filter-stopped cages inside a controlled ventilated rack with access to food and water ad libitum. They were examined daily for clinical signs, distress, decreased physical activity and weighed 3 times a week.

4) Cell xenografts

Human breast cancer xenografts from MDA-MB-231-luc cells were established in NIH nude rats. We performed forepaw injection, so that 15 rats were anaesthetized by inhalation of $1.5 \%$ isoflurane with air (Isoflo ${ }^{\circledR}$, AXIENCE S.A.S, France) and inoculated by $2 \times 10^{6}$ tumor cells in $10 \mu \mathrm{L}$ PBS into the forepaw. The rats received pre-treatment with $10 \mathrm{mg} / \mathrm{kg}$ of busulfan 2 days before cell injection, so that immunodeficiency of rats was improved.

5) Bioluminescence Imaging

BLI was performed twice a week until the end of the study (Day 28) using an IVIS-Lumina II (Perkin Elmer, France). Each rat was IP injected with $20 \mathrm{mg}$ luciferin potassium salt (Promega, France). Rats anesthetized by $1.5 \%$ isoflurane were placed on a thermostatically controlled heating pad $\left(37^{\circ} \mathrm{C}\right)$ during imaging. Acquisition binning and duration were set depending on tumor activity. Signal intensity was quantified as the total flux (photons/seconds) within ROIs drawn manually around the tumor area using Living Image 4.4 software (Perkin Elmer, France).

6) Estimation of the number of tumor cells present in the SLN

A calibration curve was determined from known amounts of cells (500, 1000, $5000,10,000 \& 50,000$ cells) either deposited on the skin or subcutaneously injected. The injected volume was $100 \mu \mathrm{L}$ of culture medium supplemented with luciferin potassium salt $(300 \mu \mathrm{g} / \mathrm{mL})$. Immediately after luciferin addition, the cell suspension was deposited or injected, and then BLI was performed $5 \mathrm{mi}-$ nutes later.

7) Near Infrared Fluorescence Imaging

NIRF was performed in vivo and ex vivo on excised specimens using an IVIS-Lumina II (Perkin Elmer, France) operated according to the spectral unmixing mode. Rats selected from BLI were IV injected with $20 \mathrm{nmol}$ of IntegriSense680

(Perkin Elmer, France). Animals anesthetized by 1.5\% isoflurane were placed on a 
thermostatically controlled heating pad in supine or lateral position. Signal intensity was quantified as the total radiant efficiency ([photons/seconds $] /\left[\mu \mathrm{W} \cdot \mathrm{cm}^{-1}\right]$ ) within ROIs drawn manually around LN area using Living Image 4.4 software (Perkin Elmer, France).

8) Assessment of fluorescence quenching

For these experiments, $50 \mu \mathrm{L}$ of Blue Patente V (0.5 nmol, Guerbet, France), IntegriSense680 (0.5 nmol, Perkin Elmer, France), ICG (129 nmol, SERB France), or $50 \mu \mathrm{L}$ of a mix of the two dyes (BPV + IntegriSense 680 or BPV + ICG) were injected in the forepaw of the rat, and then axillary LNs were resected 15 min after injection, so that NIRF was performed on excised LNs.

9) Sacrifice and organ removal

Rats under anesthesia were sacrificed by lethal IV injection of pentobarbital (Ceva Santé Animale, Libourne, France) and LNs were collected from each animal for immediate ex vivo assessments.

10) Statistical analysis

Statistical analysis was performed using Graph Pad Prism software 5.0 (Graph Pad, USA). Statistical analysis was performed with the Student's unpaired $t$ test.

\section{Results}

1) Assessments of the fluorescence quenching, and wash-out kinetics in lymph nodes from healthy rats:

There was no statistical difference in the fluorescence intensities quantified on ex vivo resected LNs after forepaw injection of IntegriSense680 versus BPV + IntegriSense680 (Figure 1(A)). There was no statistical difference in the fluorescence intensities quantified on $e x$ vivo resected LNs after forepaw injection of ICG versus BPV + ICG (Figure 1(B)). Such an absence of quenching by BPV was also observed at the time a very low amount of IntegriSense 680 was injected.

After forepaw injection of IntegriSense680, the dye diffuses mainly through the lymphatic ducts, then reaches LNs where it accumulates momentarily before being eliminated (Figure 2(A)). The comparison between NIRF signals in axillary and brachial LNs highlighted that the retention of the dye is greater in axillary LNs than in brachial LNs, furthermore there were no differences in signal intensities between $5 \mathrm{H} 30$ and $19 \mathrm{H}$ for these two LNs (Figure 2(B)). Following loco regional injection, the limited clearance in healthy LNs compromises a high sensitivity of detection of tumor cells in SLNs since the residual activity is about $1 \log$ higher than predicted fluorescence from 1000 - 2000 tumor cells. So, only a systemic route such as IV resulting in quasi-negligible residual activity in a healthy LN can be considered.

2) Validation of BLI quantitation:

The minimal invasion status was determined thanks to in vivo calibration experiments by the quantification of known amounts of cancer cells suspended in a biological medium in the presence of luciferin. The bioluminescence signal was stable during at least 9 minutes following injection and attenuation of photons 


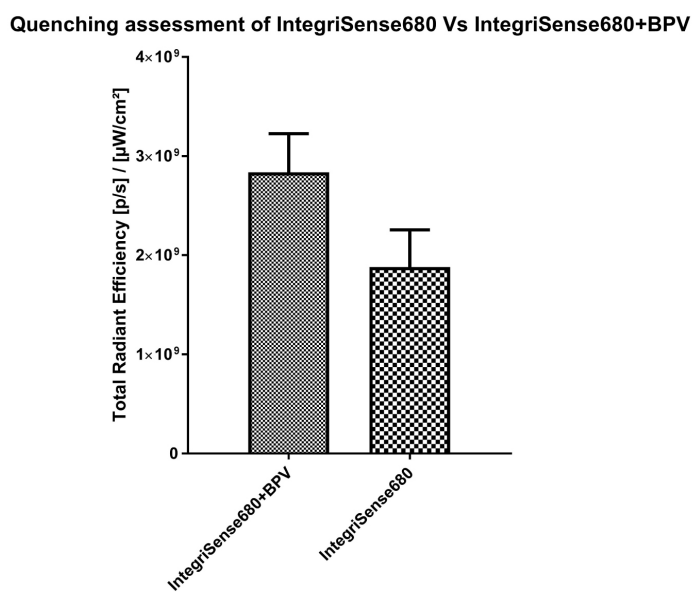

(A)

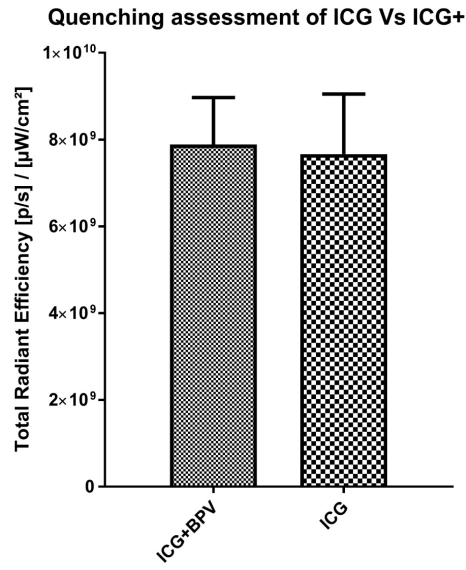

(B)

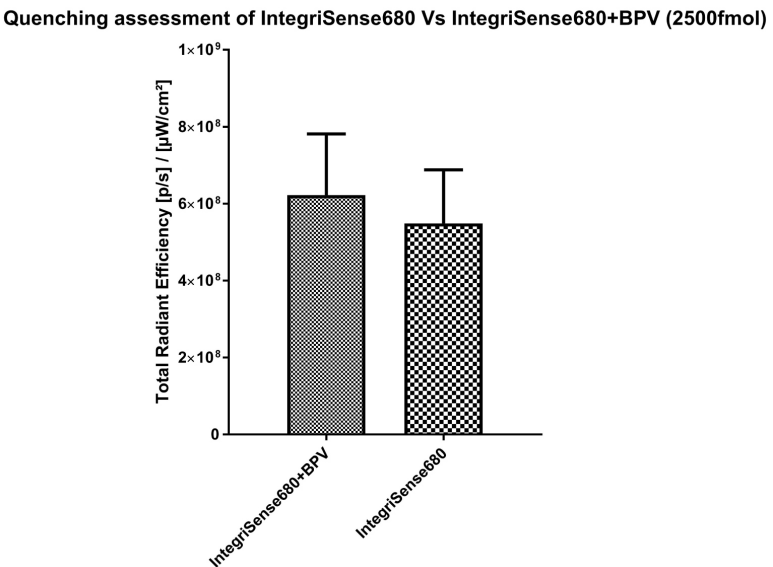

(C)

Figure 1. (A) Assessment of the fluorescence quenching of IntegriSense680 (0.5 nmol) by Patente Blue V (0.5 nmol). Quantifications performed by ex vivo NIRF on axillary LNs (n $=6 \mathrm{LN}$ per group); (B) Assessment of the fluorescence quenching of free ICG (129 nmol) by Patente Blue V $(0.5 \mathrm{nmol})$. Quantifications performed by ex vivo NIRF on axillary LNs ( $\mathrm{n}=6 \mathrm{LN}$ per group); (C) Assessment of the fluorescence quenching of IntegriSense680 (injection of $2.5 \mathrm{pmol}$ ) by Patente Blue V. Quantifications performed by ex vivo NIRF on axillary LNs ( $\mathrm{n}=6 \mathrm{LN}$ per group). 

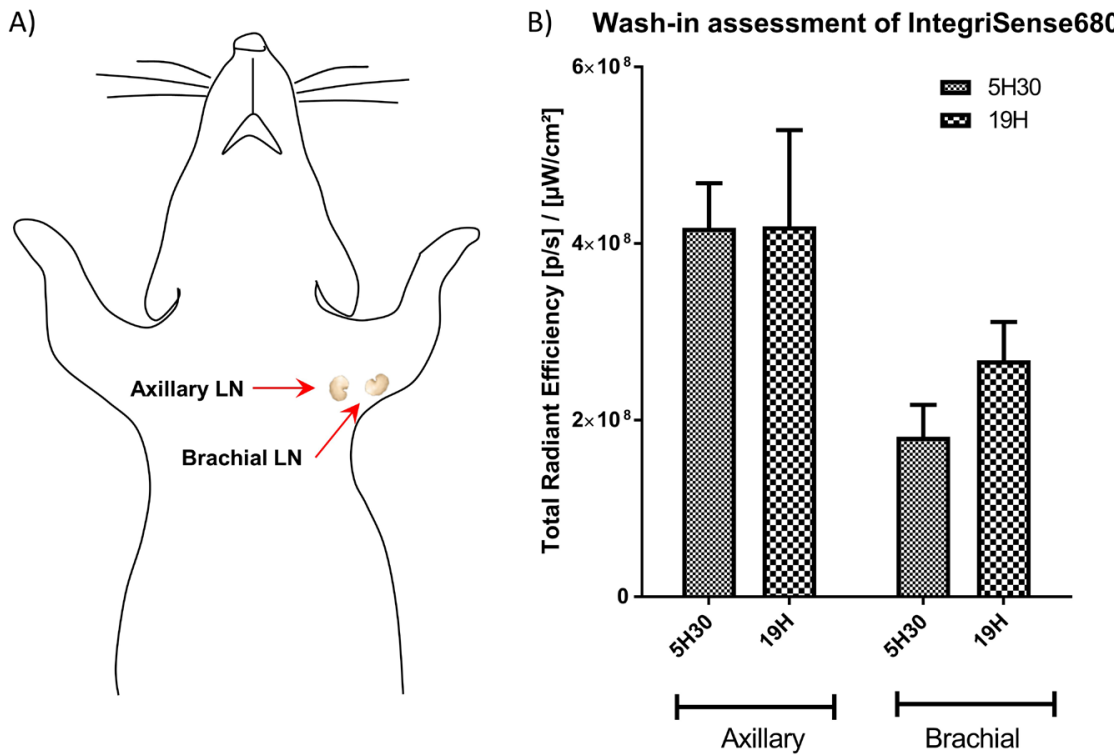

Figure 2. (A) Scheme showing the location of axial and brachial lymph nodes in the axillary area; (B) Comparison of the IntegriSense680 wash-in kinetics between axillary and brachial Lymph Nodes from rats by ex vivo NIRF quantification ( $\mathrm{n}=6 \mathrm{LN}$ per group).

by skin was about $77 \%$ (Figure 3(A)). From the known number of SC injected cells, the calibration curve of bioluminescence activity corresponds to the following equation: $Y=348 X-277556$ (Figure 3(B)) with a threshold of quantitation of about 500 cells (more than 5 times higher than background activity) (Figure 3(C)).

3) Sentinel Lymph Node invasion's model:

Thanks to BLI, it was possible to confirm the minimal invasion of tumor cells in both brachial and axillary LNs in vivo (Figure 3(A)). However, we noticed that tumor cells more often tended to develop in the axillary LN as compared to the brachial LN. From the in vivo BLI calibration curve, estimation of the tumor burden was approximately 18,700 cells for the rat A (Figure 4(A)) with a brachial invaded LN whereas rat B presented about 1500 cells (Figure 4(B)) in the axillary LN.

4) $\alpha \mathrm{V} \beta 3$ and $\alpha \mathrm{V} \beta 5$ integrins targeting:

Since locoregional injection of Integrin targeting probe IntegriSense680 results in a non-complete clearance in healthy LN, this route cannot be used to detect minimal invasion. So, only IV injections of the fluorescent probe were considered. In animals with SLNs containing almost 2000 cells, based on quantitative BLI, NIRF did not allow detection of any significant fluorescent signals in vivo. However, after excision of the SLN, ex vivo NIRF associated with spectral unmixing to suppress auto-fluorescence, enables confirmation of the minimal invasion of left axillary LN by cancer cells (Figure 5, ROI 2). Overall, the fluorescence activity of the minimally invaded LN ranged from 3 to 20 times higher than the control LNs. Fluorescence images from excised LNs were all in good correspondence with the in vivo bioluminescence patterns. 


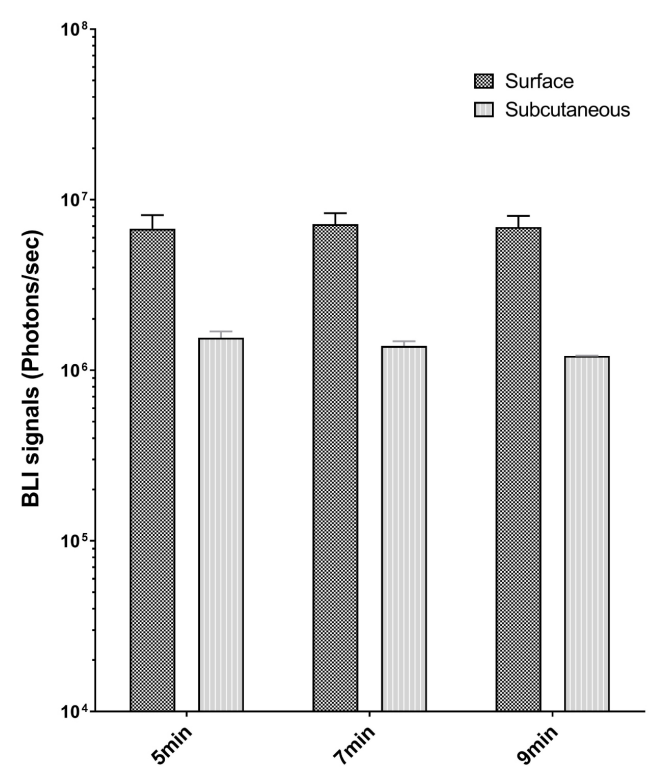

Time post luciferin injection $(\min )$

(A)

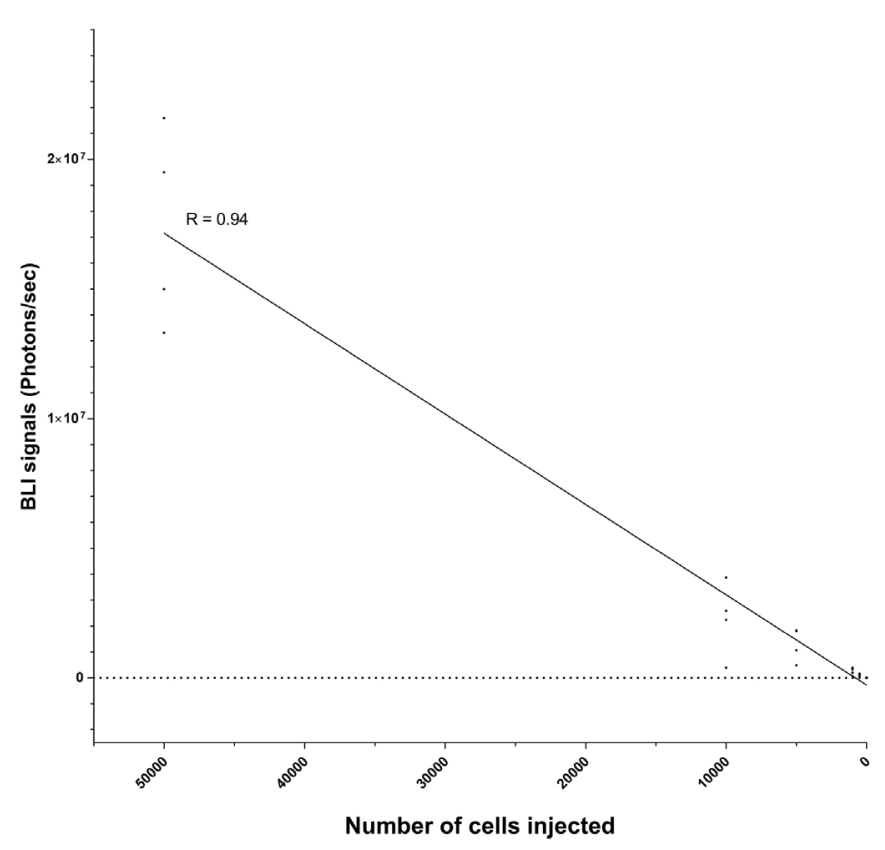

(B)

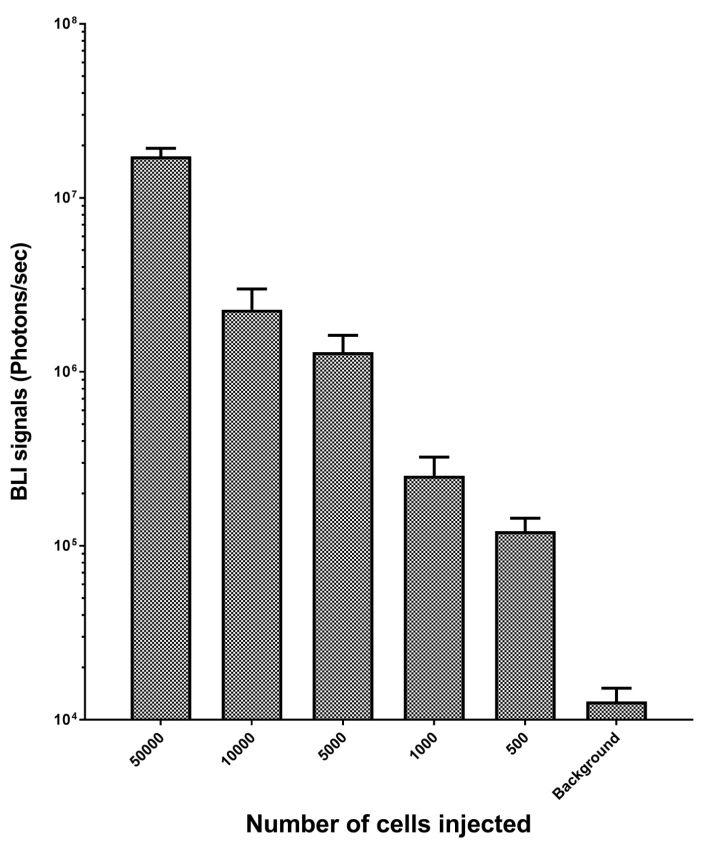

(C)

Figure 3. Validation of BLI quantification. (A) Comparison of in vivo BLI signals following subcutaneous injection or deposition of cells supplemented with substrate on the surface of the skin. ( $\mathrm{n}=2$ per condition); (B) Determination of the fitting curve for in vivo quantitation of the number of cells $(\mathrm{R}=0.94)$; (C) Assessment of BLI signal with different cell burdens following subcutaneous injection of cells supplemented with substrate. ( $n=4$ per condition).

\section{Discussion}

In clinical practice, blue dyes injected in the vicinity of primary tumors stain SLNs, so that clinicians can easily identify SLNs with the naked eyes during surgery [1]. When possible, radiolabeled colloids are co-injected allowing for the 

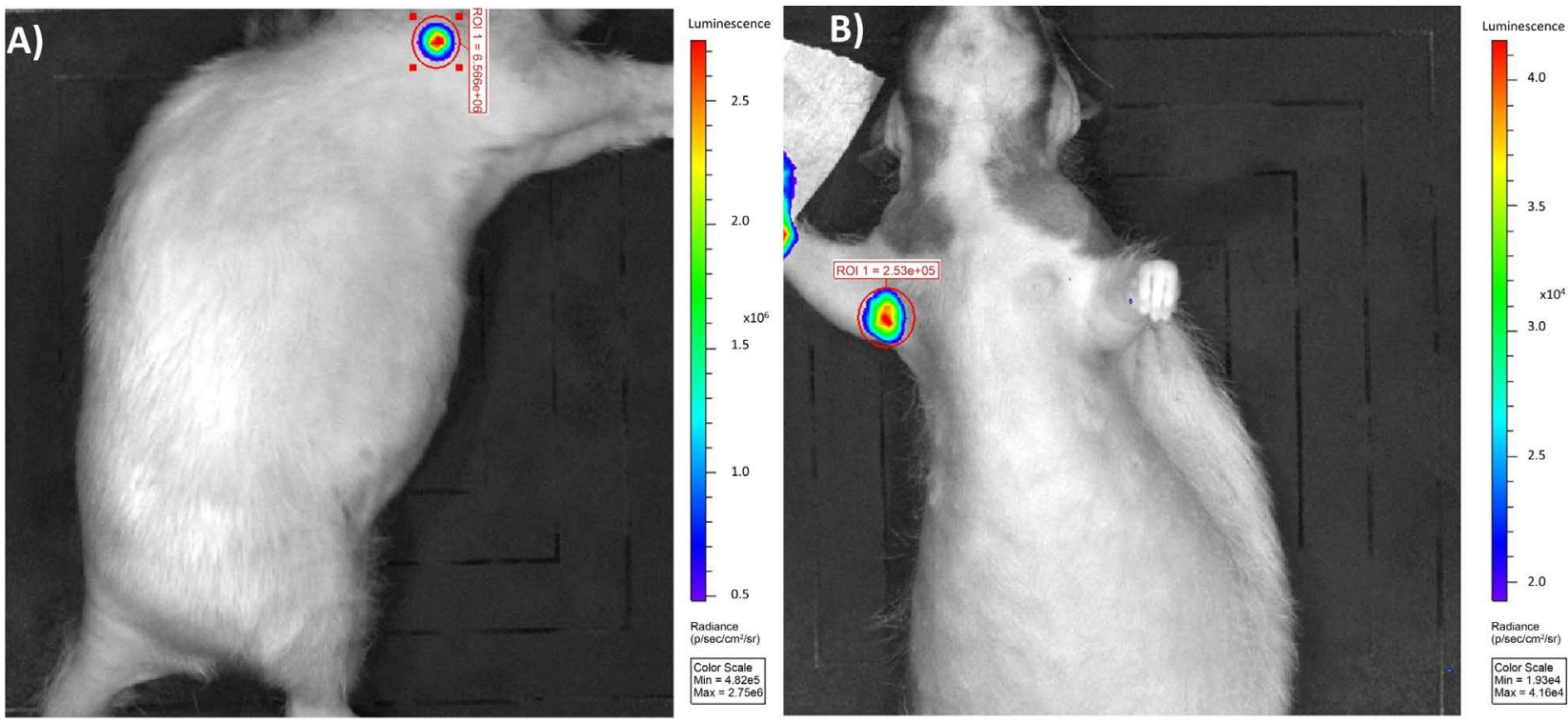

Figure 4. BLI of rats minimally invaded LN by cancer cells. To achieve more accurate quantitation, the best incidence for BLI acquisitions was anterior face for axillary LN and lateral for brachial LN. (A) BLI from right lateral side of rat A; (B) BLI from anterior side of rat B.

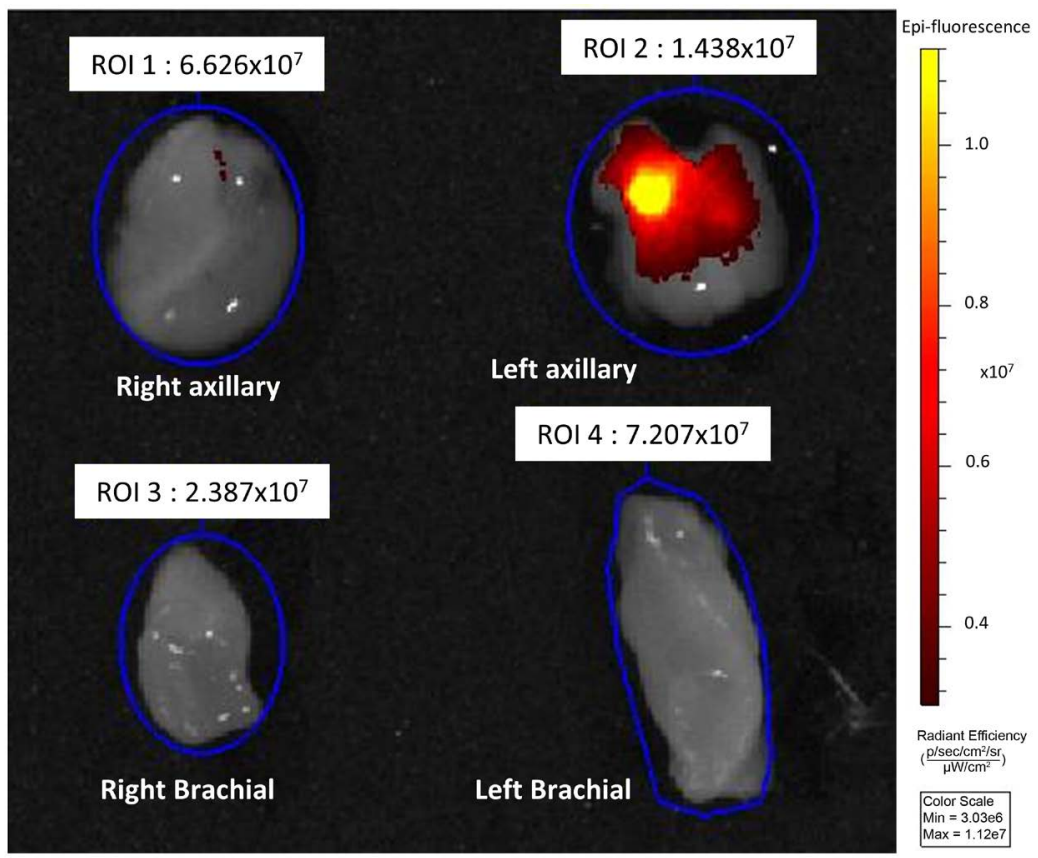

Figure 5. EX vivo NIRF identification of tumor micro-invasion in the left axillary LN after IV injection of IntegriSense680 (20 nmol). Quantifications were performed following spectral unmixing.

detection of SLN location through the skin. Considering the technical and regulatory constraints associated with the use of radioisotopes, direct imaging of the SLNs via a fluorescent probe (i.e. ICG) should be a convenient and powerful alternative. To validate the use of infrared fluorescent probes at the same time as blue dyes, we assessed the potential quenching effect when either co-injected or 
injected alone. Our results demonstrate the absence of quenching by BPV towards fluorescence of ICG and integrin targeting agents. So, fluorescence imaging is compatible with standard BPV-based surgery procedures.

When considering the simultaneous use of the two fluorescence probes (IC$\mathrm{G}_{\text {excitation }}=800 \mathrm{~nm} ; \mathrm{ICG}_{\text {emission }}=843 \mathrm{~nm}$ and IntegriSense $680_{\text {excitation }}=675$; IntegriSense $680_{\text {emission }}=693 \mathrm{~nm}$ ), optimized settings of excitation and emission filters allow for in vivo imaging of the SLN without reciprocal interferences. Due to the very lowIntegriSense680 fluorescence intensity present in micro-invaded SLNs as well as significant auto-fluorescence background, it is not possible to perform in vivo direct detection of IntegriSense680. So, ex vivo examinations on excised SLNs were performed using the spectral unmixing mode to suppress auto-fluorescence and contribution of ICG. In these conditions, the high quantum yield of fluorescence from IntegriSense 680 is favorable for the level of detection requested and allows for evidencing of micro-invasion in SLNs as low as 1500 cells.

Fluorescent dyes with an emission of around $800 \mathrm{~nm}$ are usually more suitable for intraoperative imaging due to decreased auto-fluorescence of tissues and compatibility with lighting in the operating room [15]. However, higher wavelength targeting probes suffer from a decreased quantum yield (10 to 30 times lower) resulting in insufficient intrinsic sensitivity which is no longer suitable to detect a few tumor cells even on excised specimens. Obviously it is not possible to simultaneously consider the use of ICG and a specific targeting probe emitting fluorescence in the same wavelength window. NIRF for surgical applications is a modality with great potential, helping the surgeon's vision by providing real-time acquisitions with high contrasts. Molecular probes targeting tumors, especially fluorescent MAbs and RGD-derivatives would be promising to assess the tumor invasion stage [16].

\section{Conclusion}

BLI cannot be considered in humans but it is the most sensitive imaging modality to assess minimal tumor invasion (less than 1500 cells) for oncology research in animals. It was a valuable resource to acquire a relevant model with minimal invasion. NIRF of integrins targeted dyes in an invaded LN is possible in vivo or during surgery, but its sensitivity is not sufficient enough to demonstrate micro-invasion by a direct in vivo examination. When performed on an excised LN, ex vivo high sensitivity NIRF with spectral unmixing allows for detection of the micro-invasion.

\section{Acknowledgements}

The authors thank CNRS for financial support of this study.

\section{Conflicts of Interest}

None reported. 


\section{References}

[1] Suman, B.M., Gao, S., Zhu, N., Liang, R., Gruev, V. and Achilefu, S. (2014) Real-Time Fluorescence Image-Guided Oncologic Surgery. Advances in Cancer Research, 124, 171-211. https://doi.org/10.1016/B978-0-12-411638-2.00005-7

[2] David, S. (2003) Nathanson. Insights into the Mechanisms of Lymph Node Metastasis. Cancer, 98, 413-423. https://doi.org/10.1002/cncr.11464

[3] Ohi, Y., Umekita, Y., Sagara, Y., Rai, Y., Yotsumoto, D., Matsukata, A., et al. (2012) Whole Sentinel Lymph Node Analysis by a Molecular Assay Predicts Axillary Node Status in Breast Cancer. British Journal of Cancer, 107, 1239-1243. https://doi.org/10.1038/bjc.2012.387

[4] Brambilla, T., Fiamengo, B., Tinterri, C., Testori, A., Grassi, M.M., Sciarra, A., et al. (2015) One-Step Nucleic Acid Amplification in Breast Cancer Sentinel Lymph Node: A Single Institutional Experience and a Short Review. Frontiers in Medicine (Lausanne), 2, 37. https://doi.org/10.3389/fmed.2015.00037

[5] Themelis, G., Harlaar, N.J., Kelder, W., Bart, J., Sarantopoulos, A., Van Dam, G., et al. (2011) Enhancing Surgical Vision by Using Real-Time Imaging of $\alpha \mathrm{v} \beta 3$-Integrin Targeted Near-Infrared Fluorescent Agent. Translational Research. Annals of Surgical Oncology, 18, 3506-3513. https://doi.org/10.1245/s10434-011-1664-9

[6] Lyman, G.H., Temin, S., Edge, S.N., Newman, L.A., Turner, R.R., Weaver, D.L., et al. (2014) Sentinel Lymph Node Biopsy for Patients with Early-Stage Breast Cancer. Journal of Clinical Oncology, 32, 1365-1383.

https://doi.org/10.1200/JCO.2013.54.1177

[7] Gojon, H., Fawunmi, D. and Valachis, A. (2014) Sentinel Lymph Node Biopsy in Patients with Microinvasive Breast Cancer: A Systematic Review and $\mathrm{Me}$ ta-Analysis. European Journal of Surgical Oncology, 40, 5-11. https://doi.org/10.1016/j.ejso.2013.10.020

[8] Takeuchi, H. and Yuko, K. (2015) Sentinel Lymph Node Biopsy in Gastric Cancer. Cancer Journal, 21, 21-24.

[9] Fujimura, T., Fushida, S., Tsukada, T., Kinoshita, J., Oyama, K., Miyashita, T., et al. (2015) A New Stage of Sentinel Node Navigation Surgery in Early Gastric Cancer. Gastric Cancer, 18, 210-217. https://doi.org/10.1007/s10120-014-0446-Z

[10] Handgraaf, H.J., Boogerd, L.S., Verbeek, F.P., Tummers, Q.R., Hardwick, J.C., Baeten, C.I., et al. (2016) Intraoperative Fluorescence Imaging to Localize Tumors and Sentinel Lymph Nodes in Rectal Cancer. Minimally Invasive Therapy \& Allied Technologies, 25, 48-53. https://doi.org/10.3109/13645706.2015.1042389

[11] Niu, G. and Chen, X. (2015) Lymphatic Imaging: Focus on Imaging Probes. Theranostics, 5, 686-697. https://doi.org/10.7150/thno.11862

[12] Li, L., Shiro Mori, S., Sakamoto, M., Takahashi, S. and Kodama, T. (2013) Mouse Model of Lymph Node Metastasis via Afferent Lymphatic Vessels for Development of Imaging Modalities. PLOS ONE, 8, e55797. https://doi.org/10.1371/journal.pone.0055797

[13] Bach-Gansmo, T., Danielsson, R., Saracco, A., Wilsczek, B., Bogsrud, T., Fangberget, A., et al. (2007) Integrin Receptor Imaging of Breast Cancer: A Proof-of-Concept Study to Evaluate 99mTc-NC100692. Journal of Nuclear Medicine, 47, 1434-1439.

[14] Atallah, I., Milet, C., Quatre, R., Henty, M., Reyt, E., Coll, J.L., et al. (2015) Role of Near-Infrared Fluorescence Imaging in the Resection of Metastatic Lymph Nodes in an Optimized Orthotopic Animal Model of HNSCC. European Annals of Otorhinolaryngology, Head and Neck Diseases, 132, 337-342. 
https://doi.org/10.1016/j.anorl.2015.08.022

[15] Adams, K.E., Ke, S., Kwon, S., Liang, F., Lu, Y., Hirschi, K., et al. (2007) Comparison of Visible and Near-Infrared Wavelength-Excitable Fluorescent Dyes for Molecular Imaging of Cancer. Journal of Biomedical Optics, 12, Article ID: 024017. https://doi.org/10.1117/1.2717137

[16] Desgrosellier, J.S. and Cheresh, D.A. (2010) Integrins in Cancer: Biological Implications and Therapeutic Opportunities. Nature Reviews Cancer, 10, 9-22. https://doi.org/10.1038/nrc2748 